\title{
Received Least Squares Beamforming on Ka-band Channel Model of HAP System
}

\author{
Irma Zakia, Suhartono Tjondronegoro, Iskandar, and Adit Kurniawan
}

\author{
School of Electrical Engineering and Informatics, Institut Teknologi Bandung, \\ Jl. Ganesha no. 10, Bandung 40132, Indonesia \\ irma.zakia@students.itb.ac.id, shtntjnegoro@stei.itb.ac.id, \\ iskandar@stei.itb.ac.id, adit@stei.itb.ac.id
}

\begin{abstract}
The Ka-band channel model ofHigh Altitude Platform (HAP) for high speed train is modeled as a flat and time varying Rician process with 10-25 dB Rician factor. In order to compensate for the time varying fading channel and considering the fact that the Line-of-Sight (LOS) component is dominant, adaptive beamforming with the leastsquares (LS) approach is employed at the receiver. Theoretical analysis of the LS algorithm is performed for very large Rician factor in the high Signal to Noise Ratio (SNR) regime. In particular, the analysis is with respect to the estimated autocorrelation matrix of the received vector and the beamforming weight. It is shown that, the estimated autocorrelation matrix of the received vector resembles the autocorrelation matrix of the array steering response. The presented analysis are then verified by Monte Carlo simulations.
\end{abstract}

Keywords: least-squares (LS), High Altitude Platform (HAP), Ka-band, beamforming, doppler

\section{Introduction}

As the high requirement for wireless services is inevitable, solutions for developing another wireless platform has risen. One of the services which has attracted extensive work and research recently is the requirement of providing data connections on high speed trains from existing platforms [1][2][3] as well as the emerging HAP [4][5].

Existing internet connectivity on high speed trains is given by a combination [5] of terrestrial, like UMTS, WiMAX [3], and satellite platforms. If LOS link is available, satellite system is employed in order to achieve high data rates, whereas when the train travels into deep tunnels, terrestrial system is provided to render the possibility of connectivity loss to a minimum. Accordingly, the data rate is influenced by satellite performance, which is limited to 2 Mbps [6]. In contrast, the emerging HAP platform aims at the delivery of up to $120 \mathrm{Mbps}$ data rate for trains travelling until $300 \mathrm{kmph}$, under the CAPANINA project [4][7]. Furthermore, HAP serves high speed train with lower infrastructure cost if compared to the existing terrestrial railway communication, thanks to its possible $200 \mathrm{~km}$ diameter coverage. If compared to a $36000 \mathrm{~km}$ orbiting satellite, delay propagation is of no issue on HAP.

The frequency operation of the downlink from HAP to high speed trains is Ka-band. The channel in this band is mainly LOS, whereas the nonLOS (NLOS) channel component which reflects the short-term fading, is modeled by at most two rays, each originating from different cluster [7]. In this paper, it is assumed that the railways are mostly located in rural areas. This yields the channel model consisting of a LOS link and a single ray with time indifferentiable path w.r.t. the LOS [7]. Overall, the channel is flat with Rician distributed envelope.

Since the vehicle involved is moving with high speed, a major problem is the dopplereffect which creates a time varying channel.In rural areas, moderate to high Rician factor is assumed [7], thus the received signal is mainly concerned with doppler shift which translates to a rotation of the received signal constellation. Nevertheless, if thisdoppler shifted signal is left uncompensated, a worst Bit Error Rate (BER) results [4][8].

Received: October $8^{\text {th }}$, 2013. Accepted: December $21^{\text {st }}, 2013$ 
If thedoppler shift isestimated [9][10][11], the channel mean becomes adeterministic constant parameter. Hence, the time-varying channel can be compensated by existing schemes which operate on Rician channel with constant mean, such as [12][13].But these schemes then depend on the doppler shift estimator performance. Besides, the channel is not only affected bydoppler shift, but also doppler spread for lower Rician factor[14]. The estimation of simply the doppler shift is insufficient, thus, tracking the time varying channel is required.

The physical layer adopted in this research resembles the IEEE 802.16 single carrier for Fixed Wireless Access (FWA) as proposed in [4]. This yields the channel as slow fading. With the slow fading assumption, tracking the time varying channel can be accomplished by adaptive filters, one of which is adaptive filter with the LS approach [4]. In particular, the filter is implemented in spatial domain by forming antenna arrays at the receiver. This results in LS beamforming, for which the implementation is carried out as recursive LS (RLS). The tracking is performed on the Decision Directed (DD) mode, after an initial convergence is achieved on the Data Aided (DA) one.

The contribution of this paper is on thetheoretical analysis of theLS algorithm for theKaband channel model specified in [7].Specifically, the estimated received vector autocorrelation matrix and the beam forming weight analysis are carried out in the high SNR regime for very large Rican factor. It is shown that, the estimated autocorrelation matrix of the received vector resembles the autocorrelation matrix of the array steering. Moreover, it is revealed that the beam forming weight is proportional to the array steering response, yet influenced by the Doppler effect due to the train velocity. The presented analysis are then verified by Monte Carlo simulations.

The rest of thepaper is organized as follows. Section 2 describes the transmission systems, whichcomprises the channel model and the transceiver structure. In Section 3, the LS beamforming algorithm as well as the theoretical analysis of the resulting beamf orming weight for very large Ricianfactor in the high SNR regime are presented. Afterwards, Section 4 deals with the time domain channel magnitude, estimated autocorrelation matrix of the received vector, and the beam forming weight power response obtained from Monte Carlo simulations. Furthermore, agreements of thelast two parameters to the theoretical analysis are shown. Finally, Section 5 concludes the paper and suggests topic for further investigations.

\section{Transmission Systems}

The transmission systems comprise the channel model and the transceiver structure. The channel model is already specified in [7] but a simplified model is adopted here. The transceiver structure resembles the IEEE 802.16 FWA physical layer [4].

In this paper, the transmission is the downlink from HAP equipped with a single antenna to an onboard receiver which is equipped with $N$ antennas. The onboard receiver is assumed to be deployed on top of the carriage. The transmission systems between the onboard receiver and passengers inside the train are out of the research scope.

\section{A. Channel Model}

Before representing the simplified Ka-band channel model, it is important to note that the model considered is the short-term fading channel. Only a brief description considering the simplified Ka-band channel model is given here, whereas detail analysis can be found in [7]. The simplification is w.r.t. the number of antennas on HAP, which is a single one. Therefore, it is seen as Single Input Multiple Output (SIMO) communication, a special channel model case of [7].

By assumption, the train travels mainly over rural areas. This yields a single ray NLOS model, or in addition to the LOS component, the channel model is written as

$$
\boldsymbol{h}(t)=\sqrt{\frac{K_{R}}{K_{R}+1}} \boldsymbol{h}_{L O S}(t)+\sqrt{\frac{1}{K_{R}+1}} \boldsymbol{h}_{N L O S}(t)
$$


where $h(t)=\left[h_{1}(t), h_{2}(t), \ldots, h_{N}(t)\right]^{T}, \quad h_{L O S}(t)=\left[h_{L O S ; 1}(t), h_{L O S ; 2}(t), \ldots, h_{L O S ; N}(t)\right]^{T}$,

$\mathrm{h}_{\mathrm{NLOS}}(\mathrm{t})=\left[\mathrm{h}_{\mathrm{NLOS} ; 1}(\mathrm{t}), \mathrm{h}_{\mathrm{NLOS} ; 2}(\mathrm{t}), \ldots, \mathrm{h}_{\mathrm{NLOS} ; \mathrm{N}}(\mathrm{t})\right]^{\mathrm{T}}$ describe the channel vector, the LOS channel component, and the NLOS channel component respectively. Furthermore, $K_{R}$ denotes Rician factor, which expresses the power ratio of the LOS component to the NLOS component. The LOS component is deterministic time varying, whereas the NLOS is a Wide Sense Stationary (WSS) process with independent statistical property in the time and spatial domain [7].

The downlink transmission from HAP to train is illustrated geometrically in Figure 1 [7]. The train is moving with a velocity $\vartheta$ and an elevation angle of the LOS link $\phi$. Since the HAP and the train are assumed to be located on the same plane, $\phi$ and the doppler shift of the LOS component $\psi_{0}$ are equal. The remaining of this subsection examines the LOS and NLOS component briefly.

\section{A.1. LOS Component}

From (1), the LOS component stems from

$$
\boldsymbol{h}_{L O S}(t)=\boldsymbol{a}_{R X}\left(\theta_{0}\right) e^{j 2 \pi F_{D} \cos \left(\psi_{0}\right) t}
$$

where $\boldsymbol{a}_{R X}\left(\theta_{0}\right)$ is the received steering vector on Direction of Arrival (DOA) $\theta_{0}$ and $F_{D}$ is the theoretical maximum doppler frequency [16].

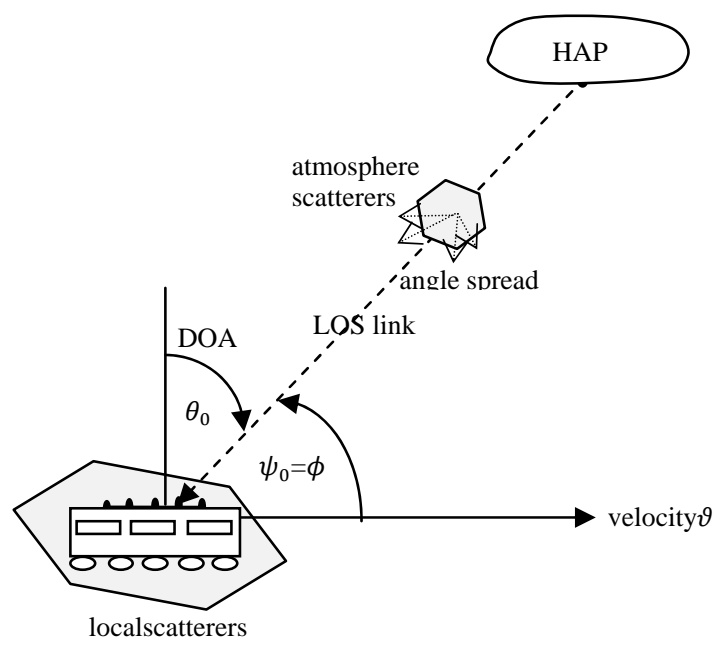

Figure 1. Downlink geometry of HAP to train channel [7]

The steering vector emerges from

$$
\boldsymbol{a}_{R X}\left(\theta_{0}\right)=\left[1, e^{j 2 \pi \sin \left(\theta_{0}\right) \frac{L}{\lambda}}, \ldots, e^{j 2 \pi(N-1) \sin \left(\theta_{0}\right) \frac{L}{\lambda}}\right]^{T}
$$

where $\lambda$ and $L$ denote the wavelength and the distance between the receiver antenna respectively. In order to avoid DOA ambiguity, the $\frac{L}{\lambda}$ value is restricted [8].

By definition, the theoretical maximum doppler frequency is expressed as [15]

$$
F_{D}=\frac{\vartheta}{c} f_{o}
$$

where $c$ and $f_{o}$ denote the speed of light and operating frequency respectively. As in [7], the Ka-band operating frequency is taken as $31 \mathrm{GHz}$. The fact that $F_{D}$ is the maximum doppler 
Irma Zakia, et al.

frequency simply acts as reference, since physically, the HAP coverage is defined for $10^{\circ} \leq$ $\psi_{0} \leq 170^{\circ}$.

\section{A.2. NLOS Component}

By the assumption that the train travels mainly on rural areas, the NLOS component is modeled as a single ray which results in a flat fading channel [7]. The scaterrers in the atmosphere produces angle spread, whereas local scaterrers surrounding the terminal induces doppler spread. The statistical description of the NLOS component is represented by the timespatial autocorrelation matrix

$$
\boldsymbol{R}(\Delta t)=E\left\{\boldsymbol{h}_{N L O S}(t) \boldsymbol{h}_{N L O S}^{H}(t-\Delta t)\right\} \in \mathbb{C}^{N, N}
$$

where $E\{$.$\} denotes expectation. The matrix \boldsymbol{R}(\Delta t)$ depends on the time difference $\Delta t$ since $\boldsymbol{h}_{N L O S}(t)$ is assumed WSS.

Following the statistical independence assumption between the doppler angle of NLOS component $\psi$ and its respective DOA $\theta$, (5) becomes

$$
\boldsymbol{R}(\Delta t)=R_{T D}(\Delta t) \boldsymbol{R}_{S D}
$$

where $R_{T D}(\Delta t)$ refers to stochastic time autocorrelation for time difference $\Delta t$ and $\boldsymbol{R}_{S D}$ is the stochastic spatial autocorrelation matrix. By definition,

$$
R_{T D}(\Delta t)=E\left\{h_{N L O S ; k}(t) h_{N L O S ; k}^{*}(t-\Delta t)\right\} \quad \forall k, k=1,2, \ldots, N
$$

where $h_{N L O S ; k}(t)$ specifies the NLOS channel $\boldsymbol{h}_{N L O S}(t)$ for the $k^{\text {th }}$ antenna. Due to the statistical independence assumption of $\psi$ and $\theta$, it is noted in (7) that $R_{T D}(\Delta t)$ is identical for all antennas, and hence a scalar. The employed time domain autocorrelation follows Clarks model [16]

$$
R_{T D}(\Delta t)=J_{0}\left(2 \pi F_{D} \Delta t\right)
$$

Since the transmitter is assumed to have a single antenna, the transmitter steering response is 1 , thus $\boldsymbol{R}_{S D}$ is defined as [7]

$$
\boldsymbol{R}_{S D}=\int_{D(\theta)} \boldsymbol{a}_{R X}(\theta) \boldsymbol{a}_{R X}^{H}(\theta) p(\theta) d \theta \in \mathbb{C}^{N, N}
$$

The matrix $\boldsymbol{R}_{S D}$ has the following physical meaning. The $(k, l)^{\text {th }}$ element of $\boldsymbol{R}_{S D}$, with $k, l=1,2, \ldots, N$, denotes NLOS correlation between the $k^{\text {th }}$ and $l^{\text {th }}$ pair. Each pair is regarded as the channel from one transmit to one received antenna. As the transmitter has single antenna, the $k^{\text {th }}$ and $l^{\text {th }}$ pairs express the channel from transmitter to received antenna $k$ and $l$ respectively.

The term $p(\theta)$ is the Probability Density Function (PDF) of the DOA $\theta$. The DOA angle spread is affected by atmospheric conditions and is assumed to follow Gaussian distribution [7]

$$
p(\theta)=\frac{1}{2 \pi \sqrt{\sigma_{\theta}^{2}}} e^{\frac{-\left(\theta-\theta_{0}\right)^{2}}{2 \sigma_{\theta}^{2}}}
$$

where $\theta_{0}$ and $\sigma_{\theta}^{2}$ denotes the DOA mean and variance respectively. The higher the variance, the channel becomes less correlated spatially. Further details on the time-spatial autocorrelation matrix of the HAP Ka-band channel is found in [7][8]. 


\section{B. Transceiver Structure}

Prior to describing the transceiver structure, the rest of this paper assumes that all variables are discrete time thus taking $t=n T_{s}$, where $n$ is integer and $T_{s}$ denotes sampling period.

The transceiver structure is visualized in Figure 2. At the transmitter, an amount of $P$ preamble symbols are transmitted for receiver training purpose. Following the preamble symbols, an amount of $D$ data symbols are transmitted. As depicted in Figure 3, each frame constitutes $P$ preamble symbols and $D$ data symbols. The amount of $P$ requireddepends on the tracking performance of the receiver algorithm, which in this case is adaptive RLS beamforming.

The transmitted symbol is thus defined as $x[n]$ in Figure 2. It can be a preamble or data

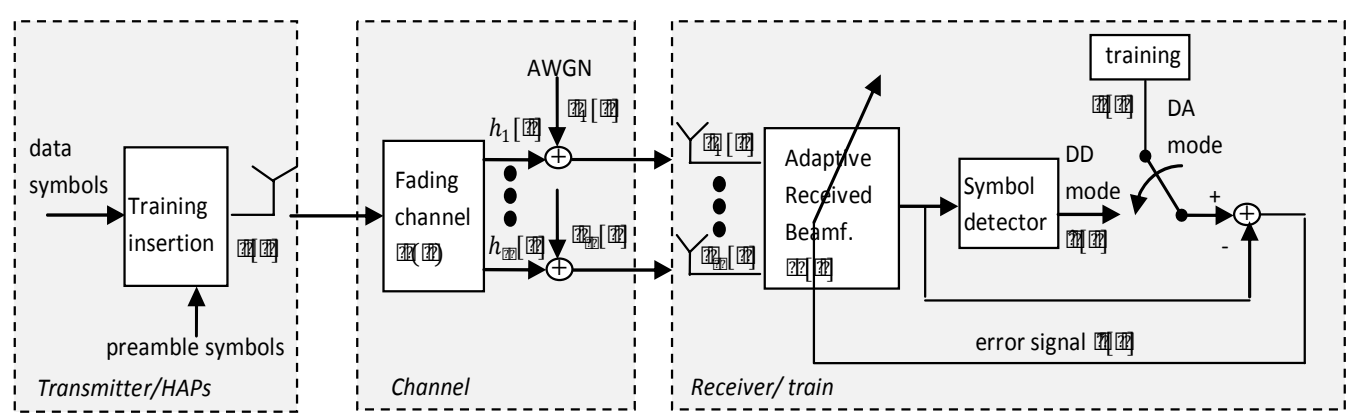

Figure 2. Transceiver Structure

symbol which depends on the position of the discrete time index $n$ of $x[n]$ on each frame, as visualized in figure 3.

On the channel, the transmitted symbol $x[n]$ experience the fading channel $\boldsymbol{h}[n]=$ $\left[h_{1}[n], h_{2}[n], \ldots, h_{N}[n]\right]^{T}$. Afterwards, Additive White Gaussian Noise (AWGN) $v[n]=$ $\left[v_{1}[n], v_{2}[n], \ldots, v_{N}[n]\right]^{T}$ is added which yields the received signal

$$
\boldsymbol{y}[n]=x[n] \boldsymbol{h}[n]+\boldsymbol{v}[n] \in \mathbb{C}^{N, 1}
$$

where $\boldsymbol{y}[n]=\left[y_{1}[n], y_{2}[n], \ldots, y_{N}[n]\right]^{T}$.

Each element of $v[n]$, denoted as $v_{k}[n], k=1,2, \ldots, N$. is i.i.d. gaussian distributed random variable. It is assumed that the noise variance $\sigma_{v}^{2}$ is equal for any antennas $k$. Denoting $E\{$.$\} as expected value, the SNR per element becomes [17]$

$$
S N R_{\text {elem }}=\frac{E\left\{\left|x[n] h_{k}[n]\right|^{2}\right\}}{\sigma_{v}^{2}}=\frac{1}{\sigma_{v}^{2}}
$$

since $x[n]$ and $h_{k}[n]$ are statistically independent, $E\left\{\left|h_{k}[n]\right|^{2}\right\}=1 \forall k=1,2, \ldots, N$, and $x[n]$ is taken from PSK constellation [4].

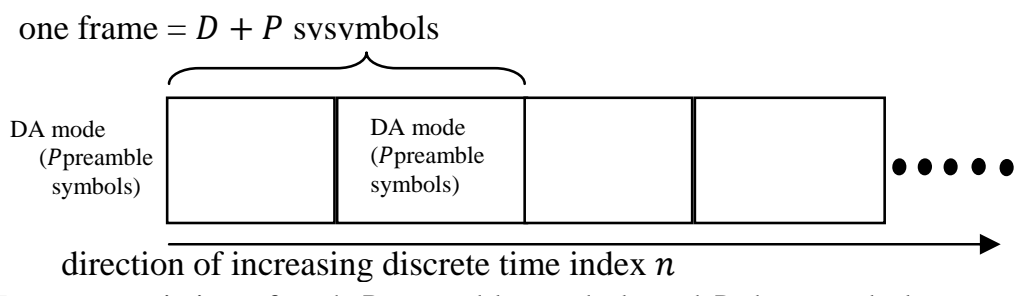

Figure 3. Frames consisting of each $P$ preamble symbols and $D$ data symbols 
Irma Zakia, et al.

Additionally, the beamforming gain can be calculated [17]

$$
G_{B F}=\frac{S N R_{\text {array }}}{S N R_{\text {elem }}}
$$

where $S N R_{\text {array }}$ is the resulting $\mathrm{SNR}$ at the beamformer output. From Figure 2, the beamformer output is expressed as

$$
\boldsymbol{y}^{T}(n) \boldsymbol{w}(n)=(\boldsymbol{h}(n) x(n)+\boldsymbol{v}(n))^{T} \boldsymbol{w}(n)
$$

where $\boldsymbol{w}[n]=\left[w_{1}[n] w_{2}[n] \ldots w_{N}[n]\right]^{T}$ is the beamforming vector.

It follows that

$$
S N R_{\text {array }}=\frac{E\left\{\left\|x(n) \boldsymbol{h}^{T}(n) \boldsymbol{w}(n)\right\|^{2}\right\}}{E\left\{\left\|\boldsymbol{v}^{T}(n) \boldsymbol{w}(n) \boldsymbol{w}^{H}(n) \boldsymbol{v}^{*}(n)\right\|^{2}\right\}}=\frac{E\left\{\boldsymbol{w}^{H}(n) \boldsymbol{h}^{*}(n) \boldsymbol{h}^{T}(n) \boldsymbol{w}(n)\right\}}{\sigma_{v}^{2} E\left\{\boldsymbol{w}^{H}(n) \boldsymbol{w}(n)\right\}}
$$

where the last equality assumes statistical independence between $\boldsymbol{v}(n)$ and $\boldsymbol{w}(n)$. Using (12), the beamforming gain becomes

$$
G_{B F}=\frac{E\left\{\boldsymbol{w}^{H}(n) \boldsymbol{h}^{*}(n) \boldsymbol{h}^{T}(n) \boldsymbol{w}(n)\right\}}{E\left\{\boldsymbol{w}^{H}(n) \boldsymbol{w}(n)\right\}}
$$

At the receiver, the channel is compensated by means of RLS adaptive beamforming. Afterwards, the transmitted data symbol are detected $\hat{x}[n]$.

From the receiver prespective, the receiver switches from DA to DD mode, and vice versa, as seen in Figure 2 and 3. Accordingly, the output of the symbol detector required for filtering is only in DD mode. The details on beamforming weight tracking $\boldsymbol{w}[n]$ is presented in Section 3 .

\section{Adaptive Least-squares Beamforming}

\section{A. Algorithm}

By assuming the channel is dominant by the LOS component, [4] proposed received beamforming, where the time varying beamforming vector $\boldsymbol{w}[n]$ is tracked by the LS algorithm. Determining the vector $\boldsymbol{w}[n]$ is pursued by minimizing the cost function [15]

$$
\varepsilon[n]=\sum_{i=1}^{n} \gamma^{n-i}\|e[i]\|^{2}
$$

where $\gamma$ indicates the forgetting factor, $0<\gamma<1$ and $e[i]$ is the error at time $i=1,2, \ldots, n$.

Before describing further, we would like to stress the fact that the LS algorithm incorporates the error $e[i]$ from time $i=1$ to $n$. Therefore, $n$ is referred as the most recent time index.

Stacking the tracking error $e[i]$ from $i=1$ to $n$ in one column results in the error vector

$$
\boldsymbol{\epsilon}[n]=[e[1], e[2], \ldots e[n]]^{T} \in \mathbb{C}^{n, 1}
$$

As a consequence, (17) is rewritten as

$$
\varepsilon[n]=\boldsymbol{\epsilon}^{H}[n] \boldsymbol{\Lambda} \boldsymbol{\epsilon}[n]
$$

where $\boldsymbol{\Lambda}=\left(\begin{array}{ccc}\gamma^{n-1} & \cdots & 0 \\ \vdots & \ddots & \vdots \\ 0 & \cdots & 1\end{array}\right)$ is a diagonal matrix with exponentially forgetting factor on its diagonal elements. Furthermore, the error vector $\epsilon[n]$ is defined as 


$$
\boldsymbol{\epsilon}[n]=\chi[n]-\boldsymbol{y}[n] \cdot \boldsymbol{w}[n]
$$

where $\chi[n] \in \mathbb{C}^{n, 1}$ is a vector with elements $x[i], i=1$ to $n$ or denoted as

$$
\chi[n]=[x[1], x[2], \ldots, x[n]]^{T}
$$

The vector $\chi[n]$ constitutes the scalar element $x[i]$ from $i=1$ to $n$. In accordance to Figure 2 , the scalar $x[n]$ is the last element of $\chi[n]$.

The matrix $\boldsymbol{y}[n]$ is found to be

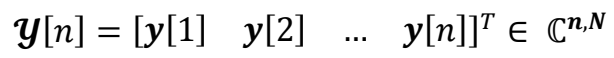

It is formed by concatenating the received signal vector $\boldsymbol{y}[i]$ from time $i=1$ to $n$ columnwise, then transposing it. In connection to Figure 2, the scalar $y_{k}[n], k=1,2, \ldots, N$ constitute $\boldsymbol{y}[n]$ in (22). Generally speaking, $\boldsymbol{y}[i]=\left[y_{1}[i], y_{2}[i], \ldots, y_{N}[i]\right]^{T}, i=1,2, \ldots, n$.

In order to track the beamforming weight $\boldsymbol{w}[n]$, training symbols are required. The mode during trainingor DA mode, proceeds until a sufficient low Mean Square Error (MSE) value results. Afterwards, the algorithm switches to DD mode. Even in LOS condition, the channel mean is time varying due to doppler shift. Accordingly, adaptive tracking of the beamforming weight is necessary. The particular steps of the RLS implementation are described in [15].

Rewriting the cost function (17) in relation to the beamforming weight

$$
\boldsymbol{w}[n]=\arg \min _{\boldsymbol{w}(n)} \boldsymbol{\epsilon}^{H}[n] \boldsymbol{\Lambda} \boldsymbol{\epsilon}[n],
$$

it is well known that the minimum is attained by orthogonal projection of $\chi[n]$ onto the space spanned by the columns of $\boldsymbol{Y}[n]$ [15]. As such, the error $\boldsymbol{\epsilon}(n)$ is orthogonal to the column space of $\boldsymbol{y}[n]$. Since the minimization (23) involves exponentially weighted error $\boldsymbol{\Lambda} \boldsymbol{\epsilon}[n]$, the weight is determined equivalently from the following

$$
\begin{aligned}
& \boldsymbol{y}^{H}[n] \boldsymbol{\Lambda} \boldsymbol{\epsilon}[n]=\mathbf{0} \\
& \boldsymbol{y}^{H}[n] \boldsymbol{\Lambda}(\chi[n]-\boldsymbol{y}[n] . \boldsymbol{w}[n])=\mathbf{0} \\
& \boldsymbol{y}^{H}[n] \boldsymbol{\Lambda} \boldsymbol{y}[n] . \boldsymbol{w}[n]=\boldsymbol{y}^{H}[n] \boldsymbol{\Lambda} \chi[n] \\
& \boldsymbol{w}[n]=\left(\boldsymbol{y}^{H}[n] \boldsymbol{\Lambda} \boldsymbol{y}[n]\right)^{-1} \boldsymbol{y}^{H}[n] \boldsymbol{\Lambda} \chi[n]
\end{aligned}
$$

As time progress, it is seen from (27) that the LS attempts to estimate the autocorrelation matrix $\boldsymbol{R}_{y y}$, as well as the crosscorrelation vector $\boldsymbol{r}_{y x}$ by time averaging. These estimates can then be defined as [15]

and

$$
\widehat{\boldsymbol{R}}_{\boldsymbol{y y}}=\boldsymbol{y}^{H}[n] \boldsymbol{\Lambda} \boldsymbol{y}[n]
$$

$$
\hat{\boldsymbol{r}}_{\boldsymbol{y x}}=\boldsymbol{y}^{H}[n] \boldsymbol{\Lambda} \chi[n]
$$

where $\widehat{\boldsymbol{R}}_{y y}$ and $\widehat{\boldsymbol{r}}_{\boldsymbol{y x}}$ indicate the deterministic autocorrelation matrix estimate and deterministi ccross correlation vector estimate respectively. This leads to the beamforming weight

$$
\boldsymbol{w}[n]=\widehat{\boldsymbol{R}}_{\boldsymbol{y} \boldsymbol{y}}^{-1} \hat{\boldsymbol{r}}_{\boldsymbol{y} \boldsymbol{x}}
$$


Irma Zakia, et al.

From the point of view of filtering, the elements of $\boldsymbol{w}[n]$ acts as the filter coefficients. The filter can be implemented in the spatial domainand executed on a single snapshot, or implemented within a tapped delay line structure (time domain implementation) which works on multiple snapshots. Since the the filter considered here is implemented in the spatial domain, the resulting correlation estimates $\left(\widehat{\boldsymbol{R}}_{\boldsymbol{y} \boldsymbol{y}}\right.$ and $\left.\widehat{\boldsymbol{r}}_{\boldsymbol{y} \boldsymbol{x}}\right)$ are correlation among different spatial antennas, not for different time..

If the filtering operation is implemented in time domain, then (22) has a completely different form [13]. In this case, (28) and (29) becomes the estimated time domain autocorrelation matrix and estimated time domain cross correlation vector respectively. Regardless of the fact that the filter weight can be implemented in spatial or time domain, the estimated autocorrelation matrix and cross correlation vector yet stem from time averaging.

\section{B. Theoretical Analysis}

The discrete-time channel model $\left(t=n T_{s}\right)$ of (1) is expressed as

$$
\boldsymbol{h}[n]=\sqrt{\frac{K_{R}}{K_{R}+1}} \boldsymbol{h}_{L O S}[n]+\sqrt{\frac{1}{K_{R}+1}} \boldsymbol{h}_{N L O S}[n]
$$

Likewise to the fading channel $\boldsymbol{h}[n]$, the discrete-time LOS channel component is indicated by $\boldsymbol{h}_{L O S}[n]=\left[h_{L O S ; 1}[n], h_{L O S ; 2}[n], \ldots, h_{L O S ; N}[n]\right]^{T}$ and its NLOS component is represented by $\boldsymbol{h}_{N L O S}[n]=\left[h_{N L O S ; 1}[n], h_{N L O S ; 2}[n], \ldots, h_{N L O S ; N}[n]\right]^{T}$.

The remaining subsection belongs to the theoretical analysis of the impact of the LS beam forming weight on the Ka-band channel. Before proceeding to the analysis, we require the transposed of the column-wise vector $\boldsymbol{h}[i]$ concatenation from $i=1$ to $n$. This yields

$$
\mathcal{H}[n]=[\boldsymbol{h}[1] \quad \boldsymbol{h}[2] \quad \ldots \quad \boldsymbol{h}[n]]^{T} \in \mathbb{C}^{n, N}(32)
$$

In general, (31) is regarded as

$$
\boldsymbol{h}[i]=\sqrt{\frac{K_{R}}{K_{R}+1}} \boldsymbol{h}_{L O S}[i]+\sqrt{\frac{1}{K_{R}+1}} \boldsymbol{h}_{N L O S}[i] \quad i=1,2, \ldots, n
$$

Then, it follows that (32)yields

$$
\mathcal{H}[n]=\sqrt{\frac{K_{R}}{K_{R}+1}} \mathcal{H}_{L O S}[n]+\sqrt{\frac{1}{K_{R}+1}} \mathcal{H}_{N L O S}[n]
$$

where

$$
\boldsymbol{H}_{L O S}[n]=\left[\boldsymbol{h}_{L O S}[1] \quad \boldsymbol{h}_{L O S}[2] \quad \ldots \quad \boldsymbol{h}_{L O S}[n]\right]^{T} \in \mathbb{C}^{n, N}
$$

and

$$
\boldsymbol{H}_{N L O S}[n]=\left[\boldsymbol{h}_{N L O S}[1] \quad \boldsymbol{h}_{N L O S}[2] \quad \ldots \quad \boldsymbol{h}_{N L O S}[n]\right]^{T} \in \mathbb{C}^{n, N}
$$

Using (34), the received signal matrix of (22) becomes

$$
\mathcal{Y}[n]=\operatorname{diag}(\chi[n]) \mathcal{H}[n]+\mathcal{V}[n]
$$

where 


$$
\operatorname{diag}(\chi[n])=\left[\begin{array}{cccc}
x[1] & 0 & \ldots & 0 \\
0 & x[2] & 0 & \vdots \\
\vdots & \ddots & \ddots & 0 \\
0 & \ldots & 0 & x[n]
\end{array}\right]
$$

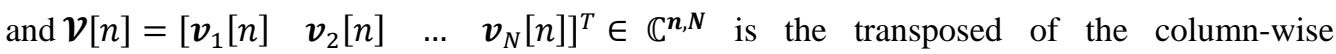
AWGN vector $v[i]$ concatenation from $i=1$ to $n$.

The Rician factor for applications supported by HAP in Ka-band is defined as 10-25 dB [7]. Hence, it is appropriate to present the LS beamforming analysis for very large Rician factor. However, for completeness of understanding, the analysis for very small Rician factor is also given.

B.1. Very Large Rician Factor

If Rician factor $K_{R}$ is very large, (34) produce

$$
\mathcal{H}[n] \approx \mathcal{H}_{\text {LOS }}[n]
$$

Inserting (35) and the discrete-time version of (2) into (39) yields

$$
\mathcal{H}[n] \approx \boldsymbol{\Psi}[n] \boldsymbol{a}_{R X}^{T}\left(\theta_{0}\right)
$$

where

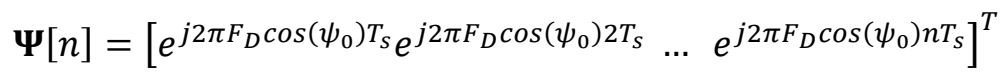

Recall that $e^{j 2 \pi F_{D} \cos \left(\psi_{0}\right) n T_{s}}$ defines the time varying doppler shift of the LOS component due to mobile velocity which results in time varying mean.

The received signal matrix for very large Rician factor emerges from

$$
\boldsymbol{y}[n] \approx \operatorname{diag}(\chi[n]) \boldsymbol{\Psi}(n) \boldsymbol{a}_{R X}^{T}\left(\theta_{0}\right)+\mathcal{V}[n]
$$

From (28), if $K_{R}$ is very large, the estimated autocorrelation matrix in the high SNR regime becomes

$$
\widehat{\boldsymbol{R}}_{\boldsymbol{y} \boldsymbol{y}} \propto \boldsymbol{a}_{R X}^{*}\left(\theta_{0}\right) \boldsymbol{a}_{R X}^{T}\left(\theta_{0}\right)
$$

This observation matches our view on $\widehat{\boldsymbol{R}}_{\boldsymbol{y} \boldsymbol{y}}$ as adeterministic spatial autocorrelation matrix estimate. Moreover, the autocorrelation matrix estimate is formed by the deterministic received steering response $\boldsymbol{a}_{R X}\left(\theta_{0}\right)$, which is different from $\boldsymbol{R}_{S D}(9)$, since the underlying process of the latter is stochastic.

Before proceeding further, it can be stated from (29) and (30) that [19]

$$
\boldsymbol{w}[n] \propto \hat{\boldsymbol{r}}_{y x}
$$

Hence, by inserting (42) to (29), it follows that

$$
\begin{aligned}
& \hat{\boldsymbol{r}}_{\boldsymbol{y} \boldsymbol{x}}=\left(\operatorname{diag}(\chi[n]) \boldsymbol{\Psi}(n) \boldsymbol{a}_{R X}^{T}\left(\theta_{0}\right)+\boldsymbol{V}[n]\right)^{H} \boldsymbol{\Lambda} \chi[n] \\
& \propto \boldsymbol{a}_{R X}^{*}\left(\theta_{0}\right) \sum_{i=1}^{n} \gamma^{n-i} e^{-j 2 \pi F_{D} \cos \left(\psi_{0}\right) i T_{S}}
\end{aligned}
$$


Irma Zakia, et al.

There are two conditions that require analysis. Firstly, if the mobile is static, i.e. $F_{D}=0$. Looking at (44) and (45), the beam forming weight attempts to compensate perfectly for the received steering response

$$
\boldsymbol{w}[n] \propto \boldsymbol{a}_{R X}^{*}\left(\theta_{0}\right)
$$

The beam forming weight is required to track the DOA of the received signal. In this case, the beam forming weight is equivalent to a spatial matched filter which maximizes the output SNR [17].

Secondly, for moving mobile, $F_{D} \neq 0$, the beamforming weight immediately yields

$$
\boldsymbol{w}[n] \propto \boldsymbol{a}_{R X}^{*}\left(\theta_{0}\right) \sum_{i=1}^{n} \gamma^{n-i} e^{-j 2 \pi F_{D} \cos \left(\psi_{0}\right) i T_{s}}
$$

Hence, besides tracking DOA, the beam forming weight requires to adjust its coefficients to the Doppler. In the CAPANINA scenario, the sampling rate $\frac{1}{T_{s}}=20 \mathrm{MHz}$, thus $F_{D} T_{S}=$ 0.00043 for a train travelling speed of $300 \mathrm{kmph}$ [4]. If each frame consists of 100 symbols, $e^{-j 2 \pi F_{D} \cos \left(\psi_{0}\right) i T_{s}}$ in (47) can be considered constant for any $i=1,2, \ldots n$. In this case, it can be stated that

$$
\boldsymbol{w}[n] \propto \boldsymbol{a}_{R X}^{*}\left(\theta_{0}\right) e^{-j 2 \pi F_{D} \cos \left(\psi_{0}\right) n T_{s}}=\boldsymbol{h}_{L O S}^{*}[n]
$$

Despite the fact that the Rician factor is very large (LOS dominant), it can be concluded that adaptive filtering is required at the receiver to track the time varying channel due to DOA and doppler shift.

\section{B.2. Very Small Rician Factor}

If Rician factor $K_{R}$ is very small, then the channel

$$
\mathcal{H}[n] \approx \mathcal{H}_{N L O S}[n],
$$

which results in the received signal matrix

$$
\boldsymbol{y}[n] \approx \operatorname{diag}(\chi[n]) \mathcal{H}_{N L O S}[n]+\mathcal{V}(n)
$$

In a similar fashion to the very large $K_{R}$ case, it is observed here that in the high SNR regime

$$
\widehat{\boldsymbol{R}}_{\boldsymbol{y y}}=\mathcal{H}_{N L O S}^{H}[n] \operatorname{diag}\left(\chi^{\boldsymbol{H}}[n]\right) \boldsymbol{\Lambda} \operatorname{diag}(\chi[n]) \mathcal{H}_{N L O S}[n]
$$

For PSK symbols, as also considers in our research, $\operatorname{diag}\left(\chi^{\boldsymbol{H}}[n]\right) \operatorname{diag}(\chi[n])=\boldsymbol{I}_{\boldsymbol{n}}$, where $\boldsymbol{I}_{\boldsymbol{n}}$ is a identity matrix with dimension $n$. Hence, by the nature of time-averaging in LS, (50) leads to

$$
\widehat{\boldsymbol{R}}_{\boldsymbol{y} \boldsymbol{y}} \propto E\left\{\boldsymbol{h}_{N L O S}[i] \boldsymbol{h}_{N L O S}^{H}[i]\right\} \text { for any } i=1,2, \ldots, n
$$

Since $\boldsymbol{h}_{N L O S}[i]$ is a stochastic process, it is observed that for very small rician factor, $\widehat{\boldsymbol{R}}_{\boldsymbol{y} \boldsymbol{y}}$ is similar to thestochastic spatial auto correlation matrix (9) or mathematically

$$
\widehat{\boldsymbol{R}}_{\boldsymbol{y} \boldsymbol{y}} \propto \boldsymbol{R}_{S D}
$$

The crosscorrelation vector emerges from 


$$
\hat{\boldsymbol{r}}_{\boldsymbol{y x}}=\mathrm{E}\left\{\left(\operatorname{diag}(\chi[n]) \boldsymbol{H}_{N L O S}[n]+\mathcal{V}[n]\right)^{H} \boldsymbol{\Lambda} \chi[n]\right\}
$$

Once again by considering (44), the beam forming weightthus becomes

$$
\boldsymbol{w}[n] \propto \sum_{i=1}^{n} \gamma^{n-i} \boldsymbol{h}_{N L O S}^{*}[i]
$$

Similarly defined to the very large Rician factor case, assuming the channel stays approximately constant for $i=1,2, \ldots, n$, (54) yields

$$
\boldsymbol{w}[n] \propto \boldsymbol{h}_{N L O S}^{*}[n]
$$

It is seen that the beamforming weight is time varying as long as the doppler exists or the mobile is moving.

By observing the results of beamforming weight at very high and very low Rician factors as given in (48) and (55) respectively, for any Rician factor and as long as the channel is assumed constant during the duration of one frame, it can be generalized that

$$
\boldsymbol{w}[n] \propto \boldsymbol{h}^{*}[n]
$$

This also means that (56) is valid for any $F_{D} T_{S}$ with an appropriate value of $P$.

\section{Simulation Results}

The simulation is performed in order to verify the theoretical analysis presented in Subsection 3.B. There are three simulation results that are presented below. As far as Ka-band applications are concerned, the Rician factors are set to $K_{R}=10 \mathrm{~dB}$ and $25 \mathrm{~dB}$, where the latter is considered very large such that it represents the theoretical analysis given in Subsection 3.B.1. However, for completeness of understanding and verifying the analysis given in Subsection 3.B.2, a very low Rician factor represented by $K_{R}=-25 \mathrm{~dB}$ is also simulated. In the simulation, QPSK modulation is used to achieve $40 \mathrm{Mbps}$ bit rate [4]. The train travels with $\vartheta=300 \mathrm{kmph}$, which results in $F_{D} T_{S}=0.00043$. The assumed SIMO system involves $N=5$ antennas at the receiver. The DOA is $\theta_{0}=30^{\circ}$.For clarity of the angle spread variance effect on the LS beam forming algorithm, two different values are taken, $\sigma_{\theta}^{2}=0.01$ and $\sigma_{\theta}^{2}=5$.

The LS algorithm is implemented as RLS beam forming. The $S N R_{\text {elem }}$ is set to10 $\mathrm{dB}$ and $20 \mathrm{~dB}$. As far as the simulation results are concerned, the RLS algorithm operates only in DA mode with $P=100$ symbols per frame. The results are then averaged over 6000 channel realizations.For the aforementioned $K_{R}$ factors and angle spread variance, the required time for the algorithm to converge for forgetting factor $\gamma=0.9$ is 35 symbols.

Firstly, the time domain channel magnitude for the first 100 symbols are given. Afterwards, the estimated autocorrelation matrix $\widehat{\boldsymbol{R}}_{y y}$ are provided. It is important to note that $\widehat{\boldsymbol{R}}_{\boldsymbol{y} \boldsymbol{y}}$ is obtained by time averaging, hence, it is a deterministic autocorrelation. Finally, the beam forming weight power response are plotted versus angle.

\section{A. Time Domain Channel Magnitude}

\section{A.1. Very LargeRician Factor}

The time domain channel magnitude of the first 100 symbols for $K_{R}=25 \mathrm{~dB}$ is depicted in Figure4. The angle spread variance assumed are $\sigma_{\theta}^{2}=0.01$ and $\sigma_{\theta}^{2}=5$. In this dominant LOS case, the effect of angle spread variance is hardly noticeable. The channel magnitude for each element of $\boldsymbol{h}[n]$ is nearly or approximately 1 . This is due to the fact that the NLOS power is only around $1 / 300$ of that of LOS, such that the overall channel power is dictated by the power of array steering response. Nevertheless, during the 100 symbols, the channel magnitude curves can be assumed to stay constant. 
Irma Zakia, et al.

\section{A.2. Moderate Rician Factor}

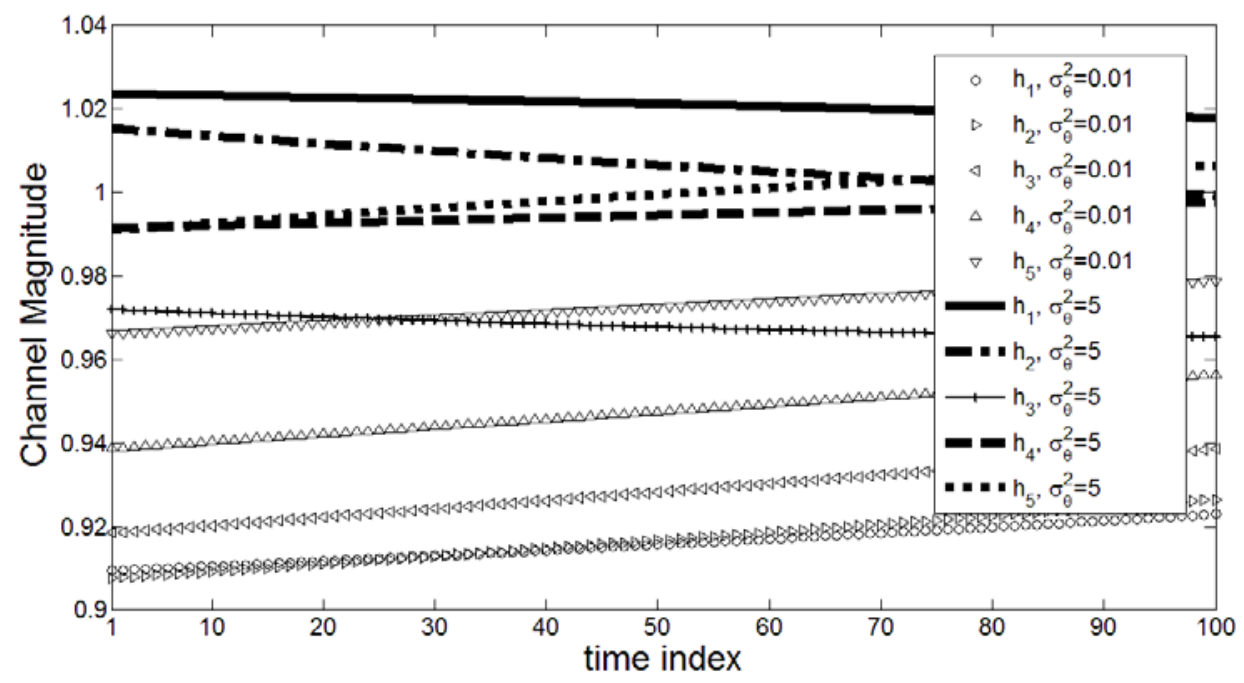

Figure 4. Time domain channel magnitude of first 100 symbols, $N=5, K_{R}=25 \mathrm{~dB}, \theta_{0}=$

$$
30^{\circ}, S N R_{\text {elem }}=10 \mathrm{~dB}, F_{D} T_{s}=0.00043 \text {. }
$$

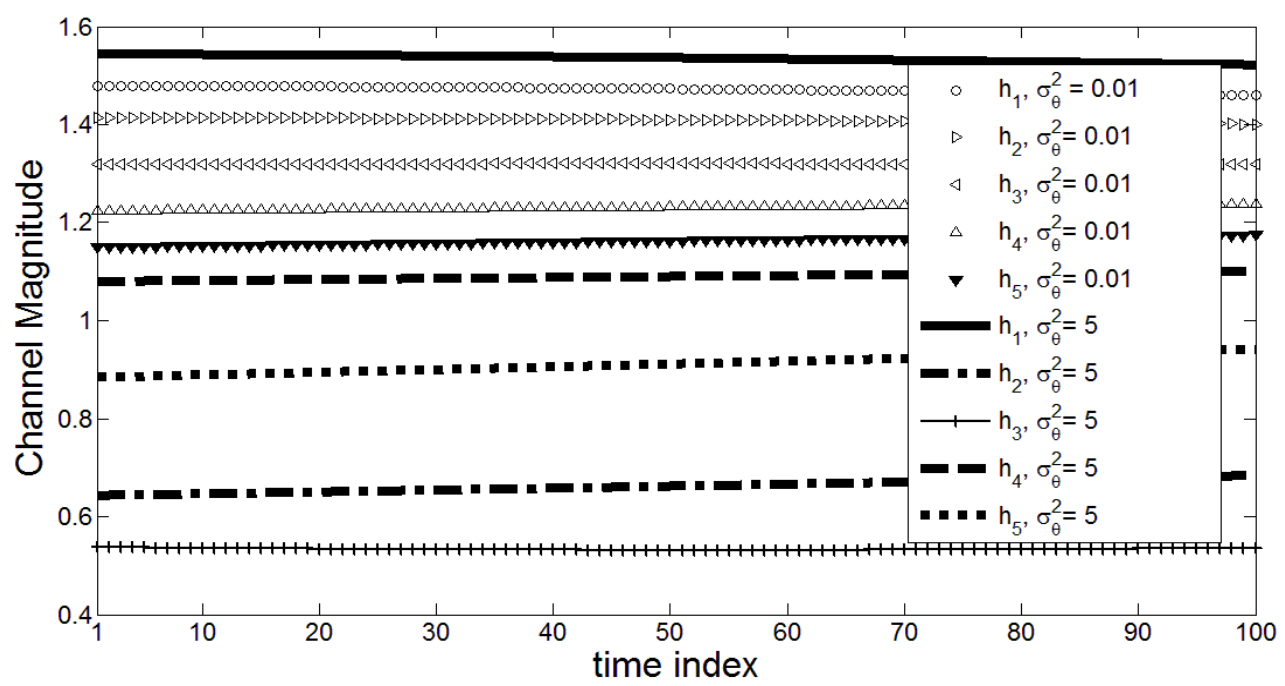

Figure 5. Time domain channel magnitude of first 100 symbols, $N=5, K_{R}=10 \mathrm{~dB}, \theta_{0}=$

$$
30^{\circ}, S N R_{\text {elem }}=10 \mathrm{~dB}, F_{D} T_{s}=0.00043 \text {. }
$$

The time domain channel magnitude of the first 100 symbols for $K_{R}=10 \mathrm{~dB}$ is depicted in Figure 5. The angle spread variance assumed are $\sigma_{\theta}^{2}=0.01$ and $\sigma_{\theta}^{2}=5$. In this moderate Rician factor, the angle spread variance influence on the channel elements magnitude is clearly seen. If $\sigma_{\theta}^{2}=0.01$, the channel is highly correlated, thus the channel elements have similar magnitude if compare to the $\sigma_{\theta}^{2}=5$ case, where the channel elements magnitude are highly distinct. Nevertheless, during the 100 symbols, the channel magnitude curves can be assumed to stay constant. 


\section{A.3. Very Small Rician Factor}

In the very small Rician factor, i.e. $K_{R}=-25 \mathrm{~dB}$, the time domain channel magnitude of the first 100 symbol is illustrated in Figure 6. If the channel is highly correlated or $\sigma_{\theta}^{2}=0.01$, the magnitude of the channel elements have a higher similarity than the $\sigma_{\theta}^{2}=5$ case. Despite this fact, it is observed that the channel magnitude curves stay approximately constant during 100 symbols.

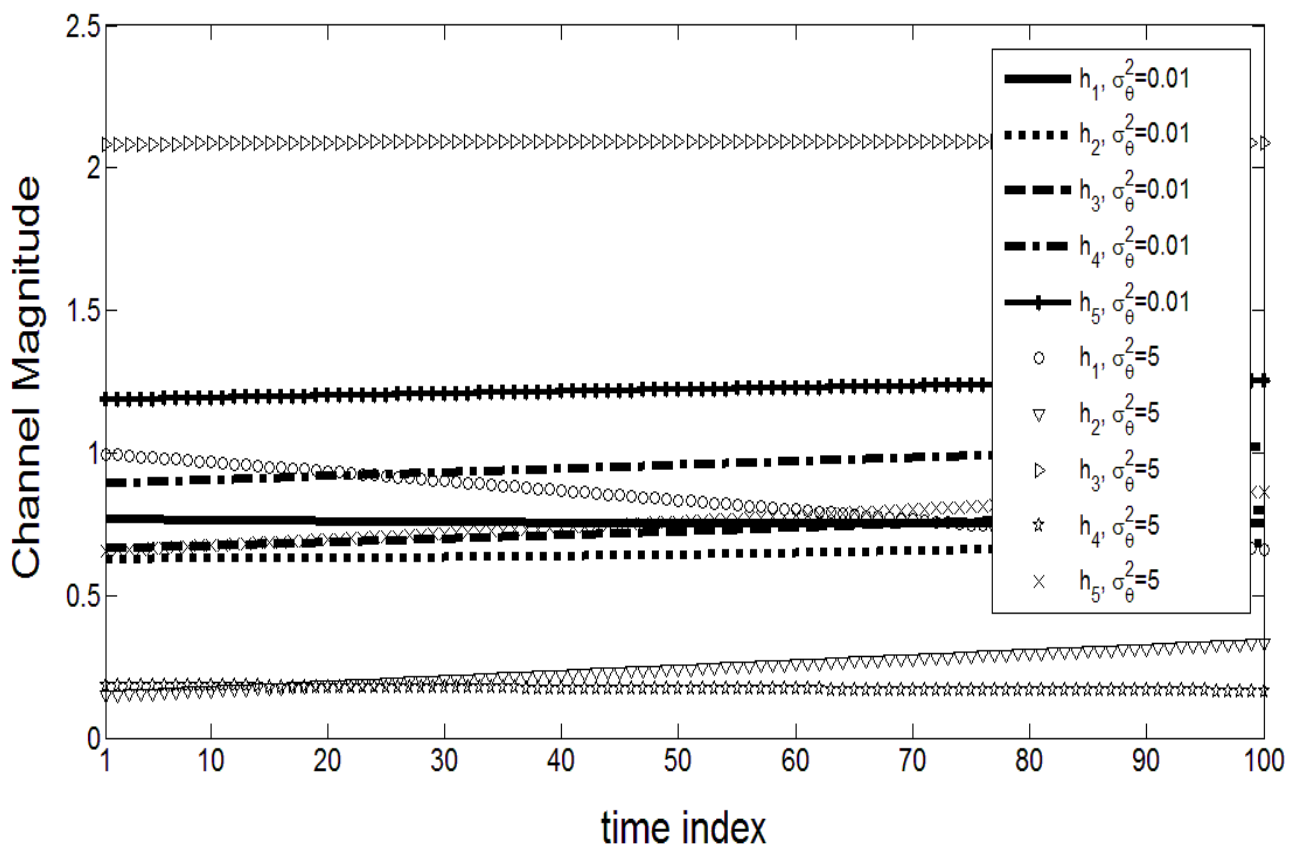

Figure 6. Time domain channel magnitude of first 100 symbols, $N=5, K_{R}=-25 \mathrm{~dB}$,

$$
\theta_{0}=30^{\circ}, S N R_{\text {elem }}=10 \mathrm{~dB}, F_{D} T_{S}=0.00043 \text {. }
$$

\section{B. Estimated Autocorrelation Matrix}

This subsection provides the spatial domain autocorrelation matrix $\boldsymbol{R}_{S D}$ for angle spread variance $\sigma_{\theta}^{2}=0.01$ and $\sigma_{\theta}^{2}=5$, with their respective magnitude plot. Furthermore, the estimated autocorrelation matrix $\widehat{\boldsymbol{R}}_{\boldsymbol{y} \boldsymbol{y}}$ magnitude is presented forvery large, moderate, and very smallRician factors. The latter is to be compared to $\boldsymbol{R}_{S D}$, which characterizes the second-order statistics of the NLOS channel.

The matrix $\boldsymbol{R}_{S D}$ is calculated from (9) and is stochastic. Its nominal DOA valueis consideredas $\theta_{0}=30^{\circ}$. For $\sigma_{\theta}^{2}=0.01$ and $\sigma_{\theta}^{2}=5$, the spatial autocorrelation matrix becomes

$R_{S D ; 0.01}=\left[\begin{array}{ccccc}1.0000+0.0000 i & 0.0070-0.9639 i & -0.8634-0.0096 i & -0.0058+0.7187 i & 0.5558-0.0030 i \\ 0.0070+0.9639 i & 1.0000+0.0000 i & 0.0070-0.9639 i & -0.8634-0.0096 i & -0.0058+0.7187 i \\ -0.8634+0.0096 i & 0.0070+0.9639 i & 1.0000+0.0000 i & 0.0070-0.9639 i & -0.8634-0.0096 i \\ -0.0058-0.7187 i & -0.8634+0.0096 i & 0.0070+0.9639 i & 1.0000+0.0000 i & 0.0070-0.9639 i \\ 0.5558+0.0030 i & -0.0058-0.7187 i & -0.8634+0.0096 i & 0.0070+0.9639 i & 1.0000+0.0000 i\end{array}\right]$

and

$$
R_{S D ; 5}=\left[\begin{array}{ccccc}
1.0000+0.0000 i & -0.3042-0.0234 i & 0.2203+0.0174 i & -0.1812-0.0145 i & 0.1575+0.0127 i \\
-0.3042+0.0234 i & 1.0000+0.0000 i & -0.3042-0.0234 i & 0.2203+0.0174 i & -0.1812-0.0145 i \\
0.2203-0.0174 i & -0.3042+0.0234 i & 1.0000+0.0000 i & -0.3042-0.0234 i & 0.2203+0.0174 i \\
-0.1812+0.0145 i & 0.2203-0.0174 i & -0.3042+0.0234 i & 1.0000+0.0000 i & -0.3042-0.0234 i \\
0.1575-0.0127 i & -0.1812+0.0145 i & 0.2203-0.0174 i & -0.3042+0.0234 i & 1.0000+0.0000 i
\end{array}\right]
$$


Irma Zakia, et al.

respectively.

The upper left part of Figure 7 and 8 illustrate the magnitude of $\boldsymbol{R}_{\boldsymbol{S D}}$ for $\sigma_{\theta}^{2}=0.01$ and $\sigma_{\theta}^{2}=5$ respectively. It is seen that as the angle spread $\sigma_{\theta}^{2}$ increases, the shape of the correlation curve is sharper, which means that correlation among different channel pairsisless.

In order to prove our theoretical analysis, i.e. (43) and (52), the estimated autocorrelation matrix $\hat{\boldsymbol{R}}_{\boldsymbol{y} \boldsymbol{y}}$ magnitude for very large, moderate, and very smallRician factors are visualized in Figure 7 and 8 . The former is concerned with low angle spread $\sigma_{\theta}^{2}=0.01$, whereas the latter with high one $\sigma_{\theta}^{2}=5$.
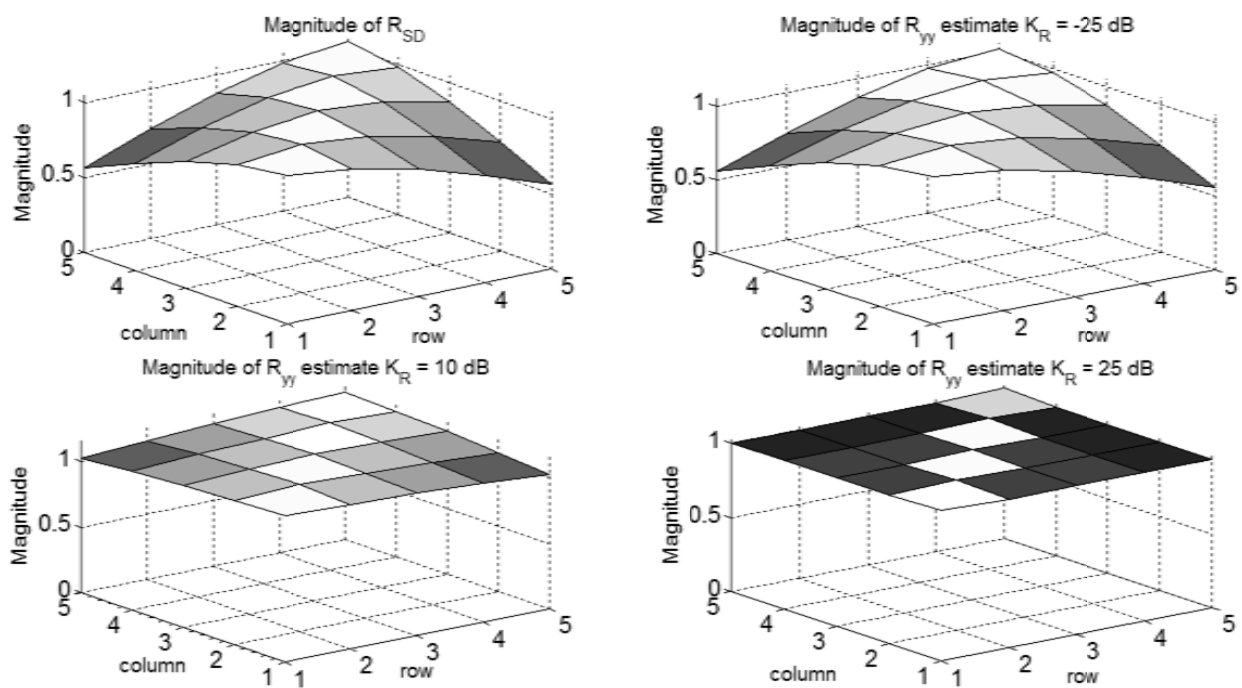

Figure 7. Comparison of $\mathrm{R}_{\mathrm{SD}}$ and $\hat{\boldsymbol{R}}_{\boldsymbol{y} \boldsymbol{y}}$ magnitude for $\mathrm{N}=5$,

$$
\theta_{0}=30^{0}, \sigma_{\theta}^{2}=0.01, \boldsymbol{S N R}_{\text {elem }}=20 \mathrm{~dB}, \boldsymbol{F}_{\boldsymbol{D}} \boldsymbol{T}_{\boldsymbol{S}}=0.00043
$$

There are two results concerning the magnitude of $\hat{\boldsymbol{R}}_{y y}$ for very large Rician factor $\left(\mathrm{K}_{\mathrm{R}}=25\right.$ $\mathrm{dB}$ ) as demonstrated by the bottom right of figure 7 and 8 . Firstly, it is observed that both bottom right figures have similar shape, which implies $\hat{\boldsymbol{R}}_{y y}$ is not affected by $\sigma_{\theta}^{2}$. Secondly, both bottom right figures indicate that the magnitude of $\hat{\boldsymbol{R}}_{\boldsymbol{y} y}$ consists of all 1's element. These two observations justify the analysis in (43). In (43), it is seen that $\hat{\boldsymbol{R}}_{\boldsymbol{y} \boldsymbol{y}}$ is independent of $\sigma_{\theta}^{2}$, and its magnitude $\left|\boldsymbol{a}_{\boldsymbol{R} X}^{*}\left(\theta_{0}\right) \boldsymbol{a}_{\boldsymbol{R X}}^{\boldsymbol{T}}\left(\theta_{0}\right)\right|$ obviously leads to an all 1's matrix element.

A different observation is found if the Rician factor is moderate $\left(K_{R}=10 \mathrm{~dB}\right)$, as visualized in the bottom left of Figure 7 and 8. The shape of both figures are influenced by their respective $\sigma_{\theta}^{2}$ value. If $\sigma_{\theta}^{2}$ is small, the channel becomes highly correlated, thus the magnitude plot of $\hat{\boldsymbol{R}}_{y \boldsymbol{y}}$ in bottom leftof Figure7 produce a fairly smooth curve, as opposed to a peaky shape if $\sigma_{\theta}^{2}$, is high (bottom left of Figure8). Additionally, the $\hat{\boldsymbol{R}}_{\boldsymbol{y} y}$ magnitude if $\mathrm{K}_{\mathrm{R}}=10 \mathrm{~dB}$, showed by the bottom left of Figure 7 and 8 , resemble closer to the $\hat{\boldsymbol{R}}_{\boldsymbol{y} \boldsymbol{y}}$ of the very large Rician factor case than to $R_{S D}$. This result is expected since $K_{R}=10 \mathrm{~dB}$ means that the LOS power is 10 times to that of NLOS, such that its $\hat{\boldsymbol{R}}_{\boldsymbol{y y}}$ magnitudeis more influenced by the auto 
correlation of the array steering response. Once again, our theoretical analysis (43) is justified by simulation.
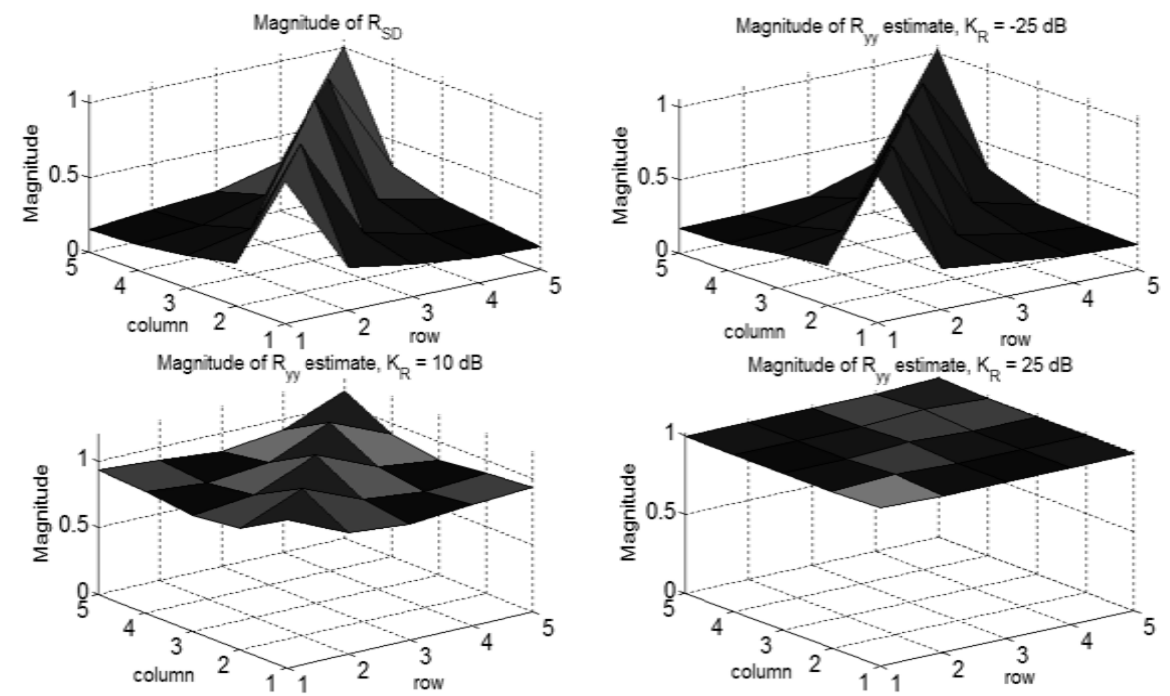

Figure 8. Comparison of $\mathrm{R}_{\mathrm{SD}}$ and $\hat{\boldsymbol{R}}_{\boldsymbol{y y}}$ magnitude for $\mathrm{N}=5$,

$$
\theta_{0=30^{0}}, \sigma_{\theta}^{2}=5, \boldsymbol{S N R}_{\text {elem }}=20 \mathbf{d B}, \boldsymbol{F}_{\boldsymbol{D}} \boldsymbol{T}_{\boldsymbol{S}}=0.00043
$$

An opposite observation is found if the Rician factor is very small $\left(K_{R}=-25 d B\right)$, as visualized in the upper right of figure 7 and 8 . Besides observing that the shape of both figures follow their respective $\sigma_{\theta}^{2}$ values very closely, the $\hat{\boldsymbol{R}}_{\boldsymbol{y} y}$ magnitude yields in a very good match to the $\mathrm{R}_{\mathrm{SD}}$ magnitude. Once again, our theoretical analysis in (52) is justified by simulation.

\section{Beamforming Weight Power Response}

In this subsection, the simulation results are divided into 3 different values of Rician factors representing very large, moderate, and very smallones. The beamforming weight power response or power response of $\mathrm{w}[\mathrm{n}]$ is plotted versus the varying angle $\theta$, where the nominal DOA value is set to $\theta_{0} 30^{\circ}$. Before proceeding to the simulation results, the beamforming weight power response is defined as [17]

$$
\boldsymbol{P}_{B F}(\theta)=10 \log _{10} E\left\{\left|\sum_{k=1}^{N} W_{k}[n] e^{j 2 \pi(k-1) \sin (\theta)} \frac{L}{\lambda}\right|^{2}\right\}
$$

For simulation purpose, the expected value is represented by averaging the inside bracket term of (57) over 6000 channel realizations.

In addition, to give more insight into the beamforming weight power response, the beamforming gain for varying Rician factor and angle spread variance are also given numerically.

\section{C.1. Very LargeRician Factor}

Depicted in Figure 9 is the beamforming weight power response for very largeRicianfactor, taken as $K_{R}=25 \mathrm{~dB}$. As stated before and emphasized in (39), very large Rician factor results in a dominant LOS channel component. This component is deterministic, hence, independent 
Irma Zakia, et al.

of the angle spread variance $\sigma_{\theta}^{2}$ employed as statistic parameter of the NLOS component.Consequently, varying the angle spread $\sigma_{\theta}^{2}$ doesn't alter the power response. Hence, the solid and dash lines, which represent low and high angle spread variance respectively, coincides.

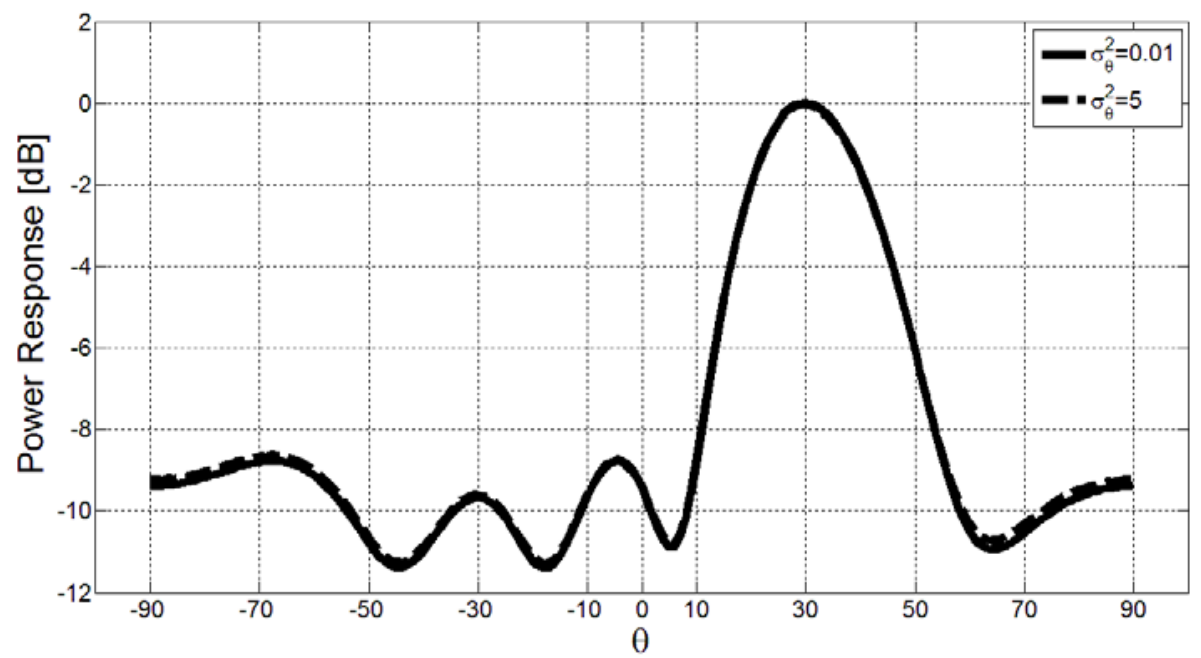

Figure 9. Beamforming weight power response for very largeRician factor, $=5, K_{R}=25 \mathrm{~dB}$,

$$
\theta_{0}=30^{\circ}, S N R_{\text {elem }}=10 \mathrm{~dB}, F_{D} T_{S}=0.00043
$$

Considering the theoretical analysis of the beamforming weight for very large Rician factor in (46) or (48), the beamforming weight is influenced by both the doppler shift and DOA. Due to the fact that Figure9 plots the power response, only the DOA can be extracted. Its power response can be understood as the power in some angle $\theta$. Since DOA $\theta_{0}=30^{\circ}$, the power response of (46) or (48) suggest high value. Therefore, the peak at $\theta=30^{\circ}$ in Figure9 shows a very good agreement with the theoretical analysis presented in Section 3.

Using (16), the beamforming gain for very large Rician factor and varying angle spread are given as

and

$$
G_{B F}\left(K_{R}=25 \mathrm{~dB}, \sigma_{\theta}^{2}=0.01\right)=3.8084
$$

$$
G_{B F}\left(K_{R}=25 \mathrm{~dB}, \sigma_{\theta}^{2}=5\right)=3.8171
$$

The beamforming gain is less than the expected gain which would be obtained from a spatial matched filter, which in a 5 receiver antennas would be $G_{B F}=5$. The resulting $G_{B F}$ here is limited by the performance of the LS algorithm such that the beamforming weight is not matched perfectly to the channel. Despite this fact, the result of $G_{B F}$ for both $\sigma_{\theta}^{2}=0.01$ and $\sigma_{\theta}^{2}=5$ are approximately equal, as is also seen on their respective power response.

\section{C.2. ModerateRician Factor}

In order to understand the effect of beamforming weight for varying Rician factors, depicted in Figure10 is the power response for moderate Ricianfactor. The chosen Rician factor is $K_{R}=10 \mathrm{~dB}$.For this value, the channel is affected by both LOS and NLOS components. Accordingly, the power response for $\sigma_{\theta}^{2}=0.01$ and $\sigma_{\theta}^{2}=5$ are distinguishable. The beamforming gain for moderate Rician factor and varying angle spread yield

and

$$
G_{B F}\left(K_{R}=10 \mathrm{~dB}, \sigma_{\theta}^{2}=0.01\right)=3.1612
$$

$$
G_{B F}\left(K_{R}=10 \mathrm{~dB}, \sigma_{\theta}^{2}=5\right)=3.9751
$$


In the respective Rician factor, the LOS is considered dominant. Nevertheless, the angle spread variance of the NLOS component yet affects the beamforming gain, which with an increasing angle spread variance translates to an increasing beamforming gain.

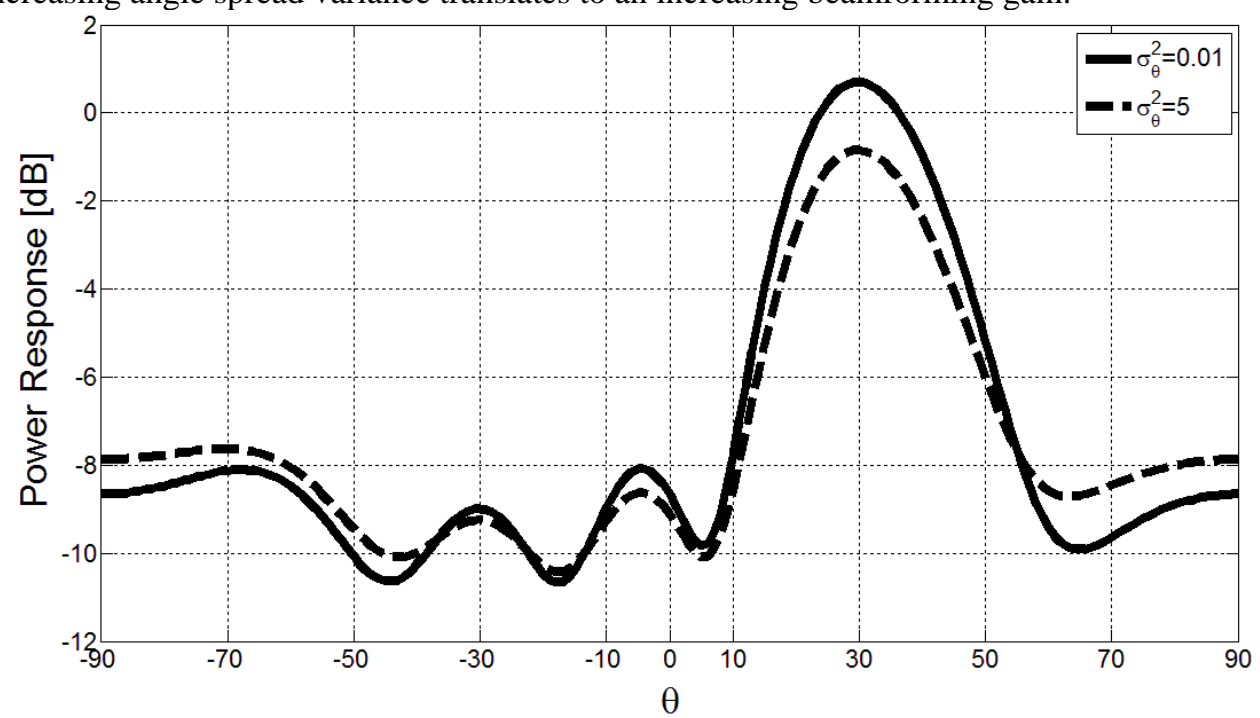

Figure 10. Beamforming weight powerresponsefor moderate Rician factor, $=5, K_{R}=10 \mathrm{~dB}$,

$$
\theta_{0}=30^{\circ}, S N R_{\text {elem }}=10 \mathrm{~dB}, F_{D} T_{S}=0.00043
$$

\section{C.3. Very Small Rician Factor}

Thebeamforming weightpower response for very small Rician factor, $K_{R}=-25 \mathrm{~dB}$, is illustrated in Figure11.From Monte Carlo simulation, if the Rician factor is very small, the beamforming weight translates into significantly different responses for different angle spread variance. Based on our analysis in (55), the beamforming weight is proportional to the random $\boldsymbol{h}_{N L O S}^{*}(n)$. This channel is statistically described by its time-spatial autocorrelation matrix (5). Additionally, the spatial autocorrelation matrix (9) is characterized by the angle spread variance. Hence, the effect of different angle spread variance on NLOS channels represented by very small Rician factor, is obvious.

Additionally observed in Figure11 is the concentration of the beamforming weight power response on the nominal DOA for $\sigma_{\theta}^{2}=0.01$. This is due to the fact that, a low angle spread variance directly translates to a high spatial autocorrelation. The resulting power response results in peak at $\theta_{0}=30^{\circ}$, though the power concentration around $30^{\circ}$ is not as high as its LOS counterpart in Figure 9.

Once again using (16), the beamforming gain for very small Rician factor and varying angle spread stem from

and

$$
G_{B F}\left(K_{R}=-25 \mathrm{~dB}, \sigma_{\theta}^{2}=0.01\right)=1.5295
$$

$$
G_{B F}\left(K_{R}=-25 \mathrm{~dB}, \sigma_{\theta}^{2}=5\right)=2.7189
$$

For very small Rician factor, the NLOS channel component dictates the weight. If $\sigma_{\theta}^{2}=5$, the channel becomes less correlated and thus the beamfoming gain is increased with the increasing of angle spread variance. 


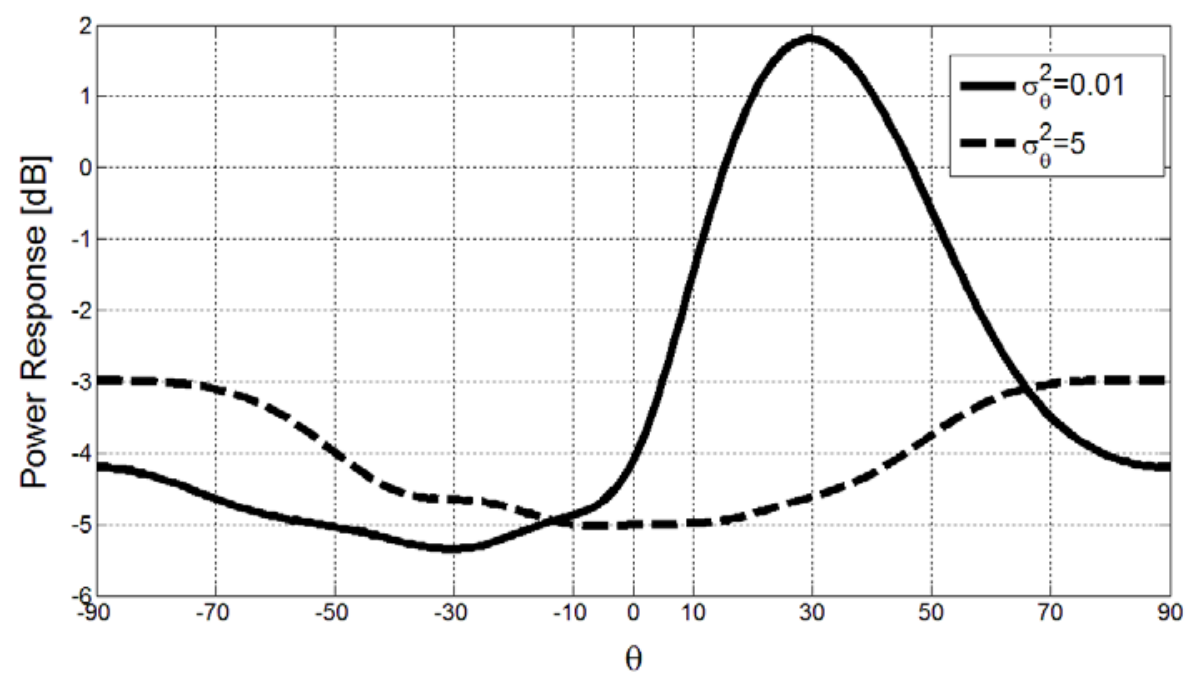

Figure 11. Beamforming weight power response for very small Rician factor, $N=5, K_{R}=$ $-25 \mathrm{~dB}, \theta_{0}=30^{\circ}, S N R_{\text {elem }}=10 \mathrm{~dB}, F_{D} T_{S}=0.00043$

We can show that for $F_{D} T_{s}=0.01$, the theoretical analysis of (48), (55), and (56) are also verified by setting a lower number of symbols per frame, i.e. $P=50$.

\section{Summary}

Theoretical analysis ofLS beamforming algorithm on the Ka-band channel model of HAP system is given for very large, and additionally, for very small Rician factors, in the high SNR regime. The analysis also considers variation in the angle spread variance of the NLOS channel component. Simulation results verify the theoretical analysis for both the estimated received vector autocorrelation matrix and beamforming weight power response.As the LS performance is influenced by the forgetting factor, it is beneficial to search foralgorithm which determines the forgetting factor based on a certain optimality criteria.Since green communications is desired, future research should consider the low SNR regime analysis and other novel receiver's algorithms with lower complexity than RLS.

\section{References}

[1] L. Liu, et. al., "Position-Based Modeling for Wireless Channel on High Speed Railway under a Viaduct at $2.35 \mathrm{GHz}$, IEEE Journal on Selected Areas in Communications, vol. 30,. No. 4, 2012.

[2] Cheng Tao, et. al., "Performance Analysis of Doppler Diversity Based on Sectorised Antenna in High-Speed Railway Communication”, Proceedings of IEEE Globecom, Dec. 2010

[3] M. Aguado, E. Jacob, M. V. Higuero, M. Berbineau and P. Saiz,"Broadband Communication in the High Mobility Scenario: the WiMAX Opportunity," in WIMAX New Developments, U. D. Dalal and Y. P. Kosta, Ed., InTech, 2009. Available from: http://www.intechopen.com/books/wimax-new-developments/broadband-communicationin-the-high-mobility-scenario-the-wimax-opportunity

[4] G. Whiteet. al., "Report on adaptive beamforming algorithms for advanced antenna types for aerial platform and ground terminals," FP6 CAPANINA Project, Doc Ref: CAP-D17WP3.3-UOY-PUB-01 www.capanina.org/documents/CAP-D17-WP3.3-UOY-PUB01.pdf, Jan. 2006.

[5] G. P. White and Y. V. Zakharov, "Data Communications to Trains FromHigh-Altitude Platforms”, IEEE Transactions On Vehicular Technology vol. 56, no. 4, July 2007. 
[6] D.T. Fokum, V. S. Frost, "A Survey on Methods for Broadband Internet Access on Trains”, IEEE Communications Surveys and Tutorials, Vol. 12, no.2, second quarter 2010.

[7] C. Spillardet. al., "Mobile link propagation aspects, channel model, and impairment mitigation techniques," FP6 CAPANINA Project, Doc Ref: CAP-D14-WP22-UOY-PUB01 www.capanina.org/documents/CAP-D14-WP22-UOY-PUB-01.pdf, April 2005.

[8] I. Zakia et.al., "Analysis of Ka-band Channel Model for High Speed Train Delivered from High Altitude Platforms”, Proceedings of International Conference on Wise-Health, Dec. 2012.

[9] H. C. So, "Adaptive algorithm for direct estimation of sinusoidal frequency", IEE Electronics Letter, vol. 36, no. 8, Apr. 2000.

[10] S. Kay, "A Fast and Accurate Single Frequency Estimator", IEEE Transactions on Acoustics, Speech, and Signal Processing, vol. 37, no. 12, Dec. 1989.

[11] G. Li, "A Stable and Efficient Adaptive Notch Filter for Direct Frequency Estimation", IEEE Transactions on Signal Processing, vol. 45, No. 8, Aug. 1997.

[12] C. Komninakis, C. Fragouli, A. Sayed, and R. D. Wesel, "Multi-Input Multi-Output Fading Channel Tracking and Equalization Using Kalman Estimation", IEEE Transactions on Signal Processing, vol. 50, No. 5, Mei 2002.

[13] S. Haykin, A. H. Sayed, J. R. Zeidler, P. Yee, and P. C. Wei.” Adaptive Tracking of Linear Time-Variant Systems by Extended RLS Algorithms”, IEEE Transactions on Signal Processing, vol. 45, No. 5, May 1997

[14] I. Zakia, S. Tjondronegoro, Iskandar, A. Kurniawan, "Performance Comparisons of Adaptive MVDR and Received LS Beamforming on the Downlink Time Varying Channel of HAP System”, in Proceedings of APCC, Aug. 2013, to be published.

[15] S. Haykin, Adaptive Filter Theory. Prentice-Hall, Inc., 1996.

[16] T. S. Rappaport, Wireless Communications. Prentice-Hall, Inc., 1998.

[17] D. G. Manolakis, V. K. Ingle, S. M. Kogon, Statistical and Adaptive Signal Processing. Mc-Graw Hill Companies Inc., 2000.

[18] A. Goldsmith, Wireless Communications, Cambridge University Press, 2005.

[19] RavirajAdve, Optimal Beamforming, Lecture Notes on Smart Antennas, University of Toronto

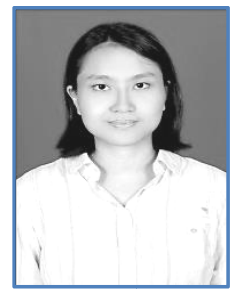

Irma Zakia received her graduate degree in electrical engineering from Institut Teknologi Bandung (ITB), Bandung, Indonesia, in 2002, and the M.Sc. degree in communications engineering from RWTH Aachen, Germany, in 2007. She is currently working towards her Ph.D. degree in ITB. Her research interests include adaptive filtering for wireless communications and information theory for multiuser communications.

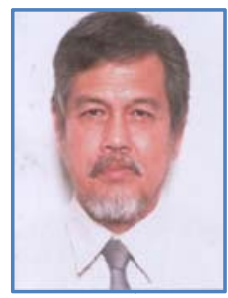

Suhartono Tjondronegoro received his Engineer's degree in Telecommunication Engineering from the Department of Electrical Engineering, Institut Teknologi Bandung (ITB), Indonesia in 1974. His Doctorate degree was obtained from the INPT (Institute Nationale Polytechnique de Toulouse), Toulouse, France, in 1987. In 1976, he joined the Department of Electrical Engineering ITB, as a teaching staff. He has completed various non-degree trainings, research activities in different countries, such as: DFVLR (Germany), Hugh Aircraft Company (USA). In the early 1990 he has been actively involved in the development of the Bachelor and Masters 
Programs on Telecommunication Engineering at ITB. He has also been actively involved in various collaboration activities with various centres in different countries, such as in European countries (TU Delft The Netherlands, Aalborg University Denmark, Unversity of Duisburg Essen Germany).He has been actively involved in Asia Link EAGER NETWIC that involves 6 universities from Asia and Europe, he was also actively involved in Erasmus Mundus Cooperation Window Mobility for Life that involves 19 universities from Asia and Europe. His current research interests include: Communication Signal Processing, Wireless Communication Systems, and Speech Coding. Dr. Suhartono Tjondronegoro is a member of IEEECommunication Society. He can be reached through shtntjnegoro@stei.itb.ac.id or shtntjnegoro@gmail.com

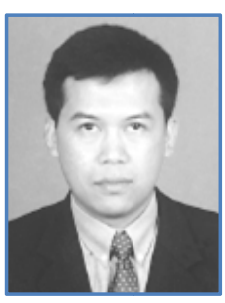

Iskandar completed his B.E. and M.E. degrees all in communications engineering from Institut Teknologi Bandung (ITB), Indonesia in 1995 and 2000, respectively. In March 2007, he received his Ph.D. degree from the Graduate School of Global Information and Telecommunication Studies (GITS), Waseda University, Japan. Since April 1997, he joined the Department of Electrical Engineering, ITB, as lecturer. His major research interests are in the areas of radio propagation, channel modelling, mobile communication, stratospheric platform, and millimetre wave band.

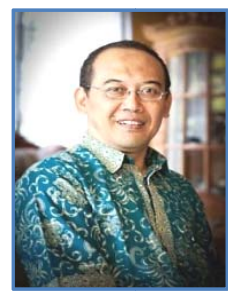

A. Kurniawan received B. Eng. in Electrical Engineering from Bandung Institut of Technology, Indonesia, in 1986. He then received M. Eng. and Ph.D in Telecommunication Engineering from the RMIT University and the University of South Australia, respectively in 1996 and 2003. He is currently Associate Professor at School of Electrical Engineering and Informatics, Bandung Institute of Technology, Indonesia. His research interestscovers the area of Antenna and Wave Propagation, and Wireless Communications. 